Vol. 6, Issue 9, September 2018

\title{
Visibility Enhancement in Surveillance and Reconnaissance Mission for Unmanned Aerial Systems
}

\author{
Ravi Tej Nulu \\ PG Student, JNTUK Kakinada
}

\begin{abstract}
Unmanned Aerial System (UAS) have become very popular these days as they are replacing human effort to carry out operations in certain areas where human beings cannot have access. They have made their way through the military as well as civilian sectors. They are also of low cost and easy to operate. During Low visibility conditions, when the UAV is flying out side of Line Of Sight (LOS), we will make use of First Person View (FPV). During such exercise images may affect with haze, blur \& noise. Dark Channel Prior method is used to remove haze from a single input image; Blurred images are handled by deploying a Wiener filter.
\end{abstract}

Keywords: UAS, LOS, FPV, Low visibility, Haze, Blur, Dark Channel Prior, Wiener filter.

\section{INTRODUCTION}

Unmanned Aerial Vehicles (UAVs) are a key component of today's Intelligence, Surveillance and Reconnaissance (ISR) missions. UAVs provide intelligence, a dynamic re-tasking capability, and real-time video imagery. UAVs are often used to get aerial video of a remote location, especially where there would be unacceptable risk to the pilot of a manned aircraft. UAVs can be equipped with high resolution still, video, and even infrared cameras. The information obtained by the UAV can be streamed back to the control centre in real time.

First Person View (FPV) is a method used to control a radio-controlled aircraft from the pilot's perspective, using a camera. It is also known as remote person view (RPV). The real-time video stream enables the remote pilot to fly the aircraft, without looking at it line of sight (LOS). Therefore, FPV is sometimes called video piloting as well. It is utilized to pilot a radio-controlled aircraft or different kinds of Unmanned Aerial Vehicles (UAVs), where the vehicle is either determined or piloted remotely from a first-person point of view by means of an on-board camera, nourished remotely to a video screen.

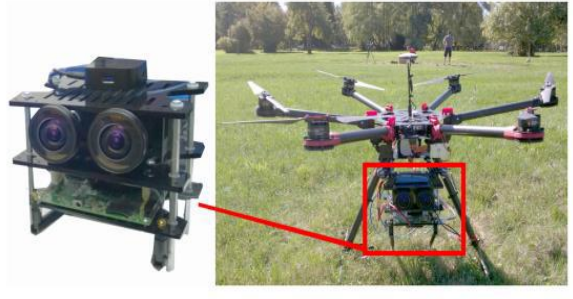

Fig: 1 UAV connected with camera

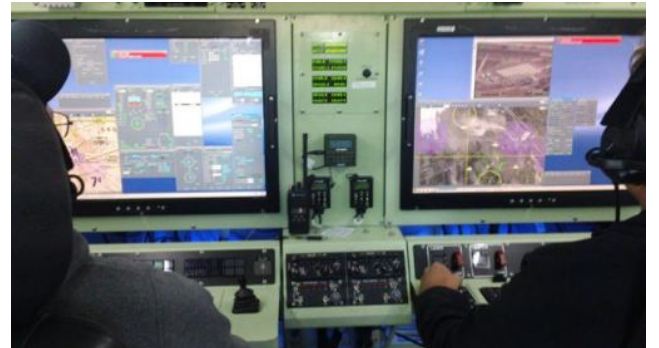

Fig: 2 Ground Control Station FPV

Maintaining a high level of Situational Awareness (SA) is one of the most critical and challenging feature of the crew. $\mathrm{SA}$ is defined as the perception of the elements in the environment within a volume of time and space. In low visibility conditions like foggy weather or during nights, blur and noise image, there are possibilities in which pilot can lose of SA.

\section{HAZE \& BLUR REMOVAL TECHNIQUES}

Images of outdoor scenes are often degraded, with fog, mist, or haze. The scene contents in such degraded images will not be easily visible. However, most of the outdoor computer vision systems, such as those used in surveillance and 


\section{International Journal of Innovative Research in Electrical, Electronics, Instrumentation and Control Engineering}

Vol. 6, Issue 9, September 2018

transportation, need to carry out meaning full scene analysis, extract useful information, and detect image features. To this end, it is imperative to remove the effects of bad weather from these images. Haze is traditionally an atmospheric phenomenon in which fog, dust, smoke, and other dry particulates (pollution) obscure the clarity of the sky. Haze removal is highly challenging due to its mathematical ambiguity, typically when the input is merely a single image, we propose a simple but effective image prior, called dark channel prior, to remove haze from a single image.

Formally, we have the following mathematical expression: For outdoor haze-free images, most patches $\Omega(\mathbf{x})$ not covering the sky should satisfy:

$$
\min _{c \in\{r, g, b\}}\left(\min _{x, \in \Omega(\mathrm{x})}\left(J_{c}\left(x^{\prime}\right)\right)\right) \approx 0 .
$$

Or more simply:

$$
J^{\text {dark }}(x) \approx 0
$$

We call this observation.

The haze imaging equation is given by: $\mathbf{I}(\mathbf{x})=\mathbf{J}(\mathbf{x}) \mathbf{t}(\mathbf{x})+\mathbf{A}(1-\mathrm{t}(\mathbf{x})) . \mathbf{x}=(x, y)$ is a $2 \mathrm{D}$ vector representing the coordinates $(x, y)$ of a pixel's position in the image. I represent the hazy image observed. $\mathbf{I}(\mathbf{x})$ is a 3D RGB vector of the colour at a pixel. $\mathbf{J}$ represents the scene radiance image. $\mathbf{J}(\mathbf{x})$ is a 3D RGB vector of the colour of the light reflected by the scene point at $\mathbf{x}$. It would be the light seen by the observer if this light were not through the haze. So we often refer to the scene radiance $\mathbf{J}$ as a haze-free image. $t$ is a map called transmission or transparency of the haze. $t(\mathbf{x})$ is a scalar in $[0,1]$. Intuitively, $t(\mathbf{x})=0$ means completely hazy and opaque, $t(\mathbf{x})=1$ means haze-free and completely clear, and $0<\mathrm{t}(\mathbf{x})<1$ means semi-transparent. $\mathbf{A}$ is the atmospheric light. It is a 3D RGB vector usually assumed to be spatially constant. It is often considered as "the colour of the atmosphere, horizon, or sky".

When the image is created, several causes happen for the image degradation such as long exposition time by camera to capture the image will result blurring due to camera vibration and motion of objects. Blur described by this equation: $\mathrm{b}$ $=\mathrm{PSF}^{*} \mathrm{c}+\mathrm{N}$,

Where: $\mathrm{b}$ the blurring image, Point Spread Function PSF, $\mathrm{c}$ the original image and $\mathrm{N}$ noise, inserted through image acquisition, that become corrupted image . To reduce blur and noise in images by using wiener filter algorithm. Basically wiener filter is used to produce an estimate of a desired or target random process by linear time-invariant filtering of an observed noisy process, assuming known stationary signal and noise spectra, and additive noise. The Wiener filter minimizes the mean square error between the estimated random process and the desired process.

Wiener filter is a filter used to produce an estimate of a desired or target random process by linear time-invariant (LTI) filtering of an observed noisy process, assuming known stationary signal and noise spectra, and additive noise. Wiener filters are usually applied in the frequency domain. Given a degraded image $x(n, m)$, one takes the Discrete Fourier Transform (DFT) to obtain $X(u, v)$. The original image spectrum is estimated by taking the product of $X(u, v)$ with the Wiener filter $G(u, v)$ :

$$
\widehat{\boldsymbol{S}}(\boldsymbol{u}, \boldsymbol{v})=\boldsymbol{G}(\boldsymbol{u}, \boldsymbol{v}) X(\boldsymbol{u}, \boldsymbol{v})
$$

The inverse DFT is then used to obtain the image estimate from its spectrum. The Wiener filter is defined in terms of these spectra.

$\boldsymbol{H}(\boldsymbol{u}, \boldsymbol{v})$ Fourier transform of the point-spread function (PSF)

$\boldsymbol{P}_{\boldsymbol{s}}(\boldsymbol{u}, \boldsymbol{v})$ Power spectrum of the signal process, obtained by taking the Fourier transform of the signal

autocorrelation

$\boldsymbol{P}_{\boldsymbol{n}}(\boldsymbol{u}, \boldsymbol{v}) \quad$ Power spectrum of the noise process, obtained by taking the

Fourier transform of the noise autocorrelation

The wiener filter is:

$$
G(u, v)=\frac{H^{*}(u, v) P_{s}(u, v)}{|H(u, v)|^{2} P_{s}(u, v)+P_{n}(u, v)}
$$


International Journal of Innovative Research in

Electrical, Electronics, Instrumentation and Control Engineering

Vol. 6, Issue 9, September 2018

\section{IMPLEMENTATION}

The below flowchart is representing the implementation of work flow

\section{FLOW CHART:}

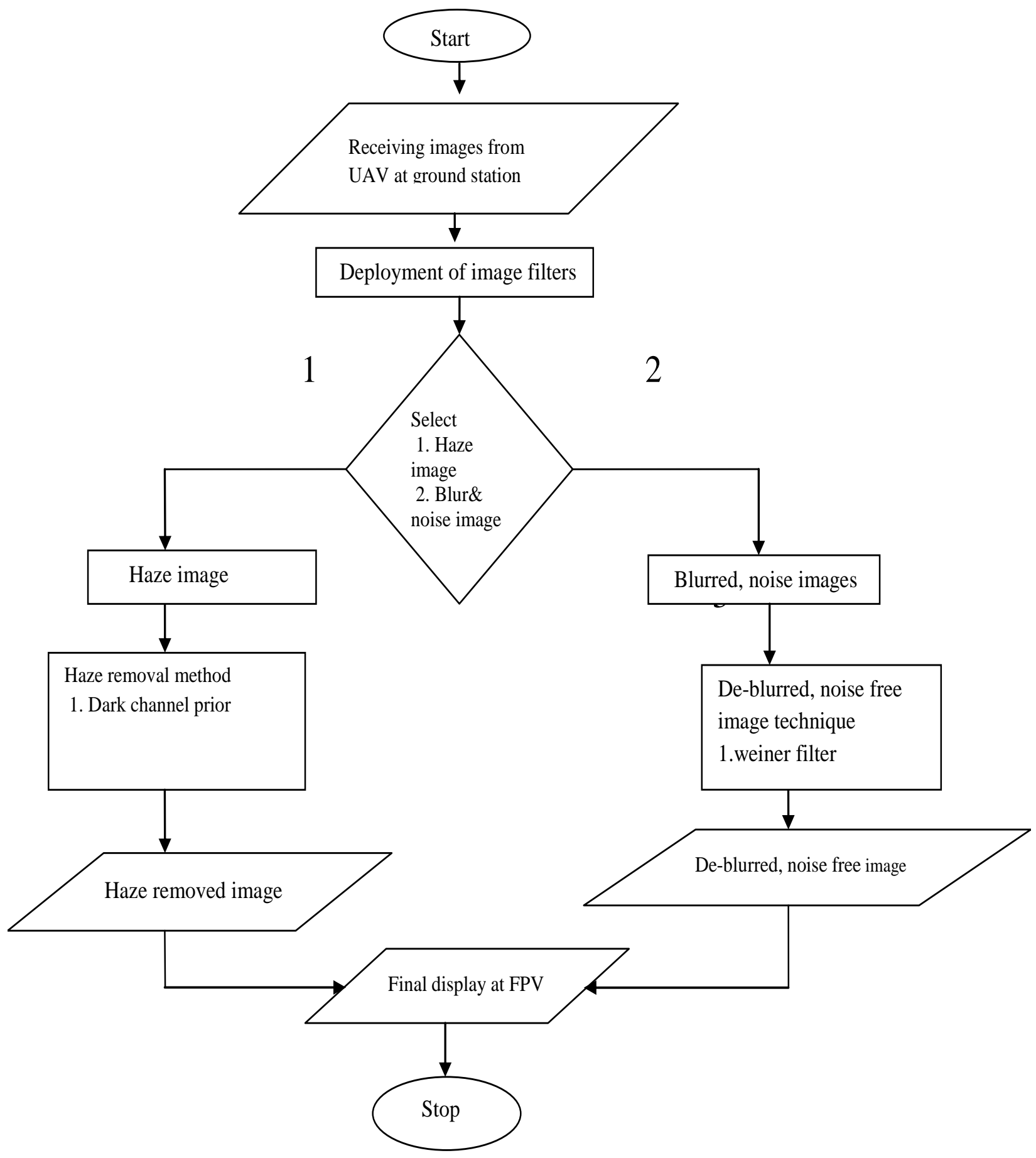




\section{International Journal of Innovative Research in Electrical, Electronics, Instrumentation and Control Engineering}

Vol. 6, Issue 9, September 2018

\section{RESULTS}

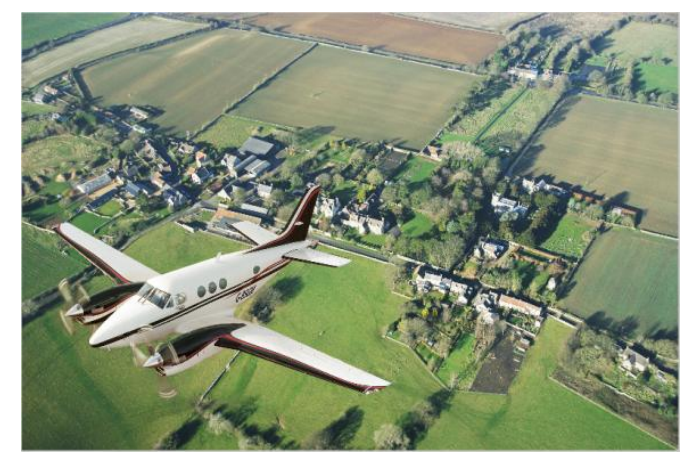

Fig: 3 Original image

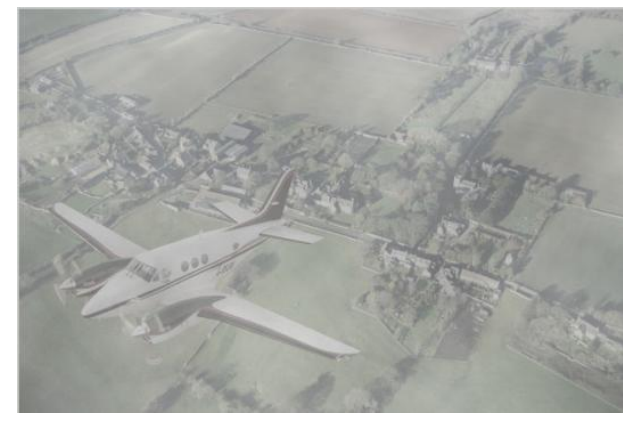

Fig: 4 Hazed image

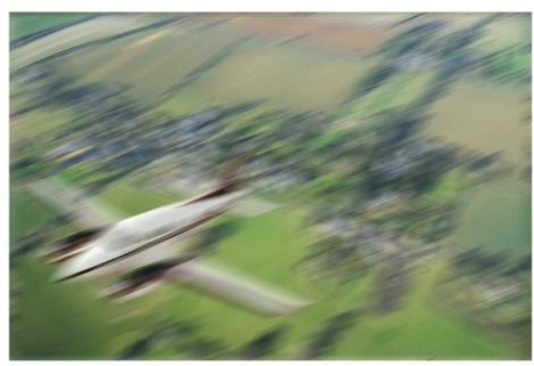

Fig: 6 Blur\& noise

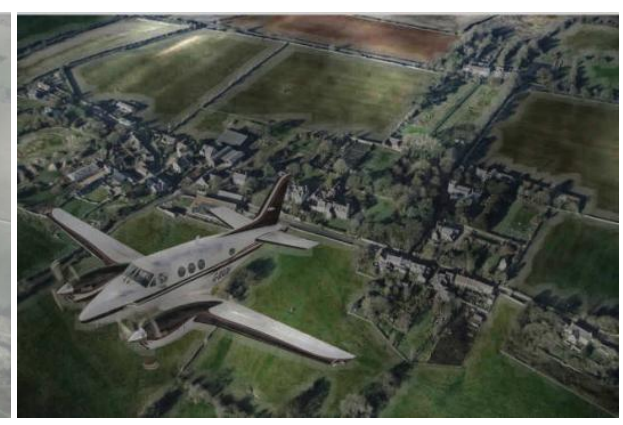

Fig: 5 De-hazed image

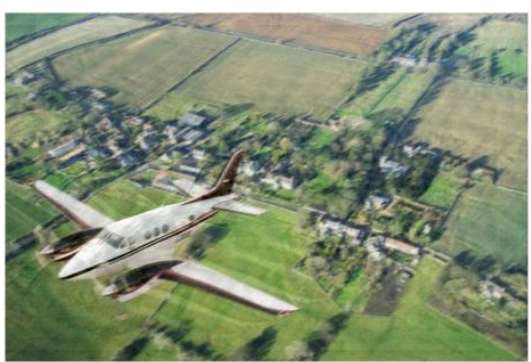

Fig: 7 De-blur\& noise free image

Above Fig: 3 is the Original image which is used as a reference image for checking the Visibility Enhancement. Fig: 4 Hazed image filled with fog, by applying dark channel prior algorithm and adjusting patch size and pad size Fig: 5 De-hazed images is obtained.

Fig: 6 is image affected by the Blur\& noise, by applying wiener filter algorithm we obtain Fig: 7 De-blur\& noise free image ,here image metrics are insignificant as it focus on perception of vision.

\section{CONCLUSION}

We propose the dark channel prior algorithm as a single image haze removal algorithm. The dark channel prior comes from an intuitive observation on outdoor haze-free images. Unlike the heuristic assumptions in previous methods, this prior is based on the image statistics, which is the result of inherent physical properties (illumination, colour, and geometry). This algorithm provides a robust estimation for each pixel, and thus solves the ambiguity of the problem. Despite of its simplicity, our haze removal algorithm based on this prior is very effective in various situations. Experiments show that our method outperforms most of the previous works. The challenge is that we need to combine the pixel-wise constraints with spatial continuities, which is usually time-consuming. 


\section{IJIREEICE}

\section{International Journal of Innovative Research in Electrical, Electronics, Instrumentation and Control Engineering}

Vol. 6, Issue 9, September 2018

Wiener filtering is the optimal trade off between the inverse filtering and noise smoothing. It can be interpreted as an inverse filtering step followed by a noise attenuation step. However, to implement the Wiener filter we have to estimate the power spectrum of the original image from the corrupted observation. This is the main task in making the Wiener filtering work well in practice. Alternatively an IR camera can remove haze in the image, but, colour information will be missing in the image and Blur in images is avoided by using gimbals for camera attached to UAV. But gimbals are too expensive. The objective is visibility enhancement by deploying image processing techniques as a low cost alternative for Haze and Blur removal.

\section{REFERENCES}

[1]. Ankita Shrivastava, Dr. Manisha Sharma "Fog Removal Techniques using Image Processing" IJIREEICE, Dept. of Electronics Engineering, Vol. 5, Issue 9, September 2017.

[2]. Robby T. Tan, Visibility in Bad Weather from a Single Image, NICTA/The Australian National University.

[3]. Kaiming He,Jian Sun, Xiaoou Tang,Single Image Haze Removal Using Dark Channel Prior, The Chinese University of Hong Kong.

[4]. Soo-Chang Pei,Tzu-Yen Lee,Effective Image Haze Removal Using Dark Channel Prior And Post-processing, Department of Electrical Engineering, National Taiwan University, Taipei, Taiwan R.O.C.

[5]. Raanan Fattal, Single Image Dehazing, Hebrew University of Jerusalem, Israel.

[6]. Naw Jacklin Nyunt, Yosuke sugiura, Tessuya Shimamura, parametric Wiener filter with parameters estimation on image power spectrum sparsity, 2017 6th International Conference on Informatics,electronics and vision.(ICIEV-ISCMHT)

[7]. Prodip Biswas, Abu Sufian Sarkar, Mohammed Mynuddin, De-blurring Images using a Wiener Filter, International Journal of Computer Applications (0975 - 8887) Volume 109 - No. 7, January 2015.

[8]. Deepak Kumar Sahu, M.P.Parsai, Different Image Fusion Techniques -A Critical Review, Department of Electronics \& Communication Engineering, Jabalpur Engineering College, Jabalpur MP, India, International Journal of Modern Engineering Research (IJMER), Vol. 2, Issue. 5, Sep.-Oct. 2012.

[9]. R.Johnson Suthakar, J.Monica Esther M.E, D.Annapoorani, F.Richard Singh Samuel, Study of Image Fusion- Techniques, Method and Applications, IJCSMC, Vol. 3, Issue. 11, November 2014

[10]. Iman Hussein AL-Qinani, De-blurring Image and Removing Noise from Medical Images for Cancerous Diseases using a Wiener Filter International Research Journal of Engineering and Technology (IRJET) 2395-0056 Volume: 04 Issue: 08 | Aug -2017.

[11]. S. Jain, and S.Goswami, "A Comparative Study of Various Image Restoration Techniques with Different Types of Blur," International Journal of Research in Computer Applications and Robotics, Vol. 3, No.11, pp: 54-60, November 2015 